\title{
ON THE DISTRIBUTIVITY AND SIMPLE CONNECTIVITY OF PLANE TOPOLOGICAL LATTICES
}

\author{
BY \\ LEE W. ANDERSON(1)
}

D. Edmundson [1] has recently constructed a counter-example to the conjecture by A. D. Wallace that every compact connected topological lattice is distributive. He discovered a subset of Euclidean three-space which, with the appropriate lattice operations, forms a nonmodular compact connected topological lattice. The main result of this paper states that if a locally compact connected subset of Euclidean two-space admits a pair of continuous lattice operations then the lattice is distributive.

1. Preliminaries. We recall that a topological lattice is a Hausdorff space, $L$, together with a pair of continuous furctions $\wedge: L \times L \rightarrow L$ and $\vee: L \times L \rightarrow L$ which satisfy the usual conditions stipulated for a lattice. As is usual we denote $\wedge(x, y)$ by $x \wedge y$ and $\vee(x, y)$ by $x \vee y$. Unless explicitly stated to the contrary, we reserve the symbols 0 and 1 to denote the unique minimal and maximal elements of a lattice, whenever they exist. A subset, $C$, of $L$ is a chain if $x \wedge y=x$ or $y$ for any pair of elements $x$ and $y$ in $C$. If $a$ and $b$ are elements of $L$ with $a \leqq b$ then $C$ is said to be a chain from $a$ to $b$ provided $C$ is a chain contained in a $\vee(b \wedge L)$ and containing both $a$ and $b$. If $A$ is a subset of $L$, we denote by $A^{*}, A^{\circ}$ and $F(A)=A^{*} \backslash A^{\circ}$ the closure, interior and boundary of $A$ respectively.

Throughout this paper, $R^{2}$ will denote the Cartesian plane with the usual topology, $S^{1}$ will denote the unit circle and $I$ will denote the closed real number interval $[0,1]$. A subset of $R^{2}$ is a simple closed curve if it is a homeomorph of $S^{1}$. We recall that Jordan's theorem [2] states that any simple closed curve cuts $R^{2}$ into exactly two components, one bounded and the other unbounded. If $C$ is a simple closed curve, we denote by $B(C)$ the bounded component of $R^{2} \backslash C$.

If $X$ is a topological space and $A$ is a subset of $X$, we denote by $H^{n}(X, A)$ the $n$-dimensional Alexander-Kolmogoroff cohomology group of $X$ modulo $A$ with coefficients in some fixed nontrivial additive abelian group. In this paper we utilize two dimension functions, namely codimension (cd) and inductive dimension (ind). For the definitions and essential theorems relating to these dimension functions the reader is referred to the work of $\mathrm{H}$. Cohen [3]. It is a pleasure to acknowledge the advice and suggestions of A. D. Wallace in the preparation of this paper.

Received by the editors August 3, 1956 and, in revised form, May 17, 1957.

(1) This work was financed by the National Science Foundation. 
2. Illustrative examples. It will be useful in the sequel to have on hand some examples of topological lattices which are topologically contained in $R^{2}$. Clearly, $R^{2}$ is a topological lattice in the usual order, i.e. $(x, y) \leqq\left(x^{\prime}, y^{\prime}\right)$ if $x \leqq x^{\prime}$ and $y \leqq y^{\prime}$. Let $L=\{(x, y) \mid x>O$ or $y<O\}$ then $L$ is a sublattice of $R^{2}$ in the usual order. We observe that $L$ contains maximal chains that are not connected.

Let $\alpha$ and $\beta$ be real numbers greater than zero. Define a relation, $\prec$, in $I \times I$ by: $(x, y) \prec\left(x^{\prime}, y^{\prime}\right)$ if, and only if, $x \leqq x^{\prime}$ and $x^{\alpha}+y^{\beta} \leqq x^{\prime \alpha}+y^{\prime \beta}$. Then $\prec$ is a partial ordering and $I \times I$ with this partial ordering is a topological lattice. We note that the center of this lattice is empty.

For each $n=1,2, \cdots$, let $L_{n}$ be the set of all pairs of real numbers, $(x, y)$, such that $n(n+1) / 2 \leqq y \leqq(n+1)(n+2) / 2$ and $n /(n+1) y+n / 2 \leqq x$ $\leqq n /(n+1) y+(n+2) / 2$. Let $L=\bigcup\left\{L_{n} \mid n=1,2, \cdots\right\}$ and define $\wedge: L \times L$ $\rightarrow L$ as follows: if $(x, y)$ and $\left(x^{\prime}, y^{\prime}\right)$ are in $L_{n}$ for some $n$, let

$$
(x, y) \wedge\left(x^{\prime}, y^{\prime}\right)=(z, w)
$$

where $z=\min \left[((n+1) / n)\left(\min \left(y, y^{\prime}\right)-y\right)+x,((n+1) / n)\left(\min \left(y, y^{\prime}\right)-y^{\prime}\right)+x^{\prime}\right.$ and $w=\min \left(y, y^{\prime}\right)$. If $(x, y) \in L_{n}$ and $\left(x^{\prime}, y^{\prime}\right) \in L_{m}$ and if $n<m$, let $(x, y)$ $\wedge\left(x^{\prime}, y^{\prime}\right)=(x, y)$. Define $\bigvee: L \times L \rightarrow L$ analogously, replacing max for min in the above formulae. Then $L$ together with the operations $\vee$ and $\wedge$ form a topological lattice.

Let $L$ be the set of pairs of real numbers, $(x, y)$, such that $0<x \leqq 1$ and $y=\sin 1 / x$ or $x=0$ and $y=0$. If $(x, y)$ and $\left(x^{\prime}, y^{\prime}\right)$ are elements of $L$, let $(x, y) \leqq\left(x^{\prime}, y^{\prime}\right)$ whenever $x \leqq x^{\prime}$. Then $L$ with this ordering is a connected, nonlocally compact, nonlocally connected, nonlocally convex topological lattice (see corollary to Theorem 4).

3. Cut points in topological lattices. If $X$ is a topological space and $p \in X$, we say that $p$ is a cut point of $X$ if $X \backslash p$ is not connected, i.e. if $X \backslash p=U \cup V$ where $U \neq \square \neq V$ and $U^{*} \cap V=\square=U \cap V^{*}$.

THEOREM 1. If $L$ is a connected topological lattice and if $p \in L$ then $p$ is a cut point of $L$ if, and only if, $p \neq 0, p \neq 1$ and $L=(p \vee L) \cup(p \wedge L)$.

Necessity. This proof is due to A. D. Wallace and was communicated to the author by him. Suppose that $L$ is connected and that $p \in L$ cuts $L$. Let $L \backslash p=U \cup V$ where $U \neq \square \neq V$ and $U^{*} \cap V=\square=U \cap V^{*}$. Let $x$ be an element of $U$ and let $y$ be an element of $V$. We will show that either $x \leqq y$ or $y \leqq x$. Suppose this is not the case, i.e., assume that $(x \wedge L) \cap(y \vee L)=\square=(x \vee L)$ $\cap(y \wedge L)$. Now $x \wedge y$ is in both $x \wedge L$ and $y \wedge L$ and so $(x \wedge L) \cap(y \wedge L) \neq \square$. Similarly $(x \vee L) \cap(y \vee L) \neq \square$. Since $x \wedge L$ and $y \wedge L$ are connected sets, $(x \wedge L) \cup(y \wedge L)$ is also connected. Moreover, $(x \wedge L) \cup(y \wedge L)$ meets both $U$ and $V$ and therefore $p \in(x \wedge L) \cup(y \wedge L)$. Similarly, we have that $p$ $\in(x \vee L) \cup(y \bigvee L)$. Thus 


$$
\begin{aligned}
p \in[(x \wedge L) \cup & (y \wedge L)] \wedge[(x \vee L) \cup(y \vee L)] \\
= & {[(x \wedge L) \cap(x \vee L)] \cup[(x \wedge L) \cap(y \vee L)] } \\
& \cup[(y \wedge L) \cap(x \vee L)] \cup[(y \wedge L) \cap(y \vee L)]=\{x\} \cup\{y\}
\end{aligned}
$$

and so $p=x$ or $p=y$. This, however, is a contradiction for $x$ and $y$ are both contained in $L \backslash p$. We therefore have that $x \leqq y$ or $y \leqq x$. Let us assume that $x \leqq y$. Now $y \wedge L$ is a connected set containing both $x$ and $y$ and so $p \in y \wedge L$. Also, $x \bigvee L$ is a connected set containing both $x$ and $y$ and therefore $p \in x \vee L$. Thus $x<p<y$ which implies that $p \neq 0$ and $p \neq 1$ and $L=(p \wedge L) \cup(p \vee L)$.

Sufficiency. Since $p \neq 0,(p \wedge L) \backslash p$ is nonvoid and since $p \neq 1,(p \vee L) \backslash p$ is also nonvoid. Now $L=(p \wedge L) \cup(p \vee L)$ and so it follows that $(p \wedge L) \backslash p$ $=L \backslash(p \bigvee L)$ and therefore, since $p \bigvee L$ is closed, $(p \wedge L) \backslash p$ is open. Likewise, $(p \backslash L) \backslash p$ is open. Thus $L \backslash p$ is the union of the disjoint open nonvoid sets $(p \wedge L) \backslash p$ and $(p \vee L) \backslash p$ which implies that $p$ cuts $L$.

A theorem in Wilder [4] states that a connected topological space $X$ is irreducibly connected about two of its elements $a$ and $b$ if, and only if, each point of $X$ distinct from $a$ and $b$ cuts $X$ into exactly two components, one containing $a$ and the other containing $b$. If $L$ is a connected topological lattice then $(x \wedge L) \backslash x$ and $(x \vee L) \backslash x$ are connected sets for all $x$ in $L$. Thus we have the

Corollary. If $L$ is a connected topological lattice with 0 and 1 then $L$ is a chain if, and only if, $L$ is irreducibly connected about 0 and 1.

3. Local convexity in topological lattices. If $A$ is a subset of a lattice $L$, let $C(A)=(A \wedge L) \cap(A \vee L)$. We say that the subset $A$ is convex if $A=C(A)$. It is clear that this condition is equivalent to: if $x$ and $z$ are elements of $A$ and if $y$ is an element of $L$ such that $x \leqq y \leqq z$ then $y$ is an element of $A$.

The proof of the following result is routine and will be omitted.

Lemma 1. If $L$ is a topological lattice and if $A$ is a subset of $L$ then

(i) $C(A)$ is open whenever $A$ is open,

(ii) $C(A)$ is connected whenever $A$ is connected.

A topological lattice $L$ is locally convex provided that whenever $x$ is an element of $L$ and $U$ is an open set containing $x$ there is an open convex set $V$ such that $x \in V \subset U$.

Lemma 2. If $L$ is a locally convex connected topological lattice and if $a \in U$, an open subset of $L$, then there exists an open convex connected subset $V$ of $L$ such that $a \in V \subset U$.

Proof. Since $L$ is locally convex, there is an open convex set $W$ such that $a \in W \subset U$. Let $M$ be the maximal connected subset of $W$ which contains $a$ We will show that $M$ is an open set. Let $y$ be an element of $M$. Now $y \wedge y=y$ $=y \bigvee y$ and $\wedge$ and $\vee$ are continuous functions hence there exists an open 
set $N$ such that $N \wedge N \subset W$ and $N \vee N \subset W$. If $x$ is an element of $N$ then $x \wedge y$ and $x \vee y$ are elements of $W$. Now $W$ is convex so that $(x \wedge y) \vee[(x \vee y) \wedge L]$ $\subset W$. However, $(x \wedge y) \vee[(x \vee y) \wedge L]$ is a connected set containing both $x$ and $y$ and therefore $x \in M$ which implies that $N \subset M$. Thus $M$ is an open connected subset of $W$. By Lemma $1, C(M)$ is open and connected and moreover, $C(M) \subset C(W)=W$. Thus if we let $V=C(M)$ we have that $V$ is an open convex connected subset of $U$ which contains $a$.

In particular, Lemma 2 implies that a locally convex connected topological lattice is locally connected. It has already been shown [5] that a locally compact connected topological lattice is locally convex so that we have the

Theorem 2. A locally compact connected topological lattice is locally connected.

A nondegenerate locally compact locally connected separable connected metrizable topological space is a Peano space in the terminology of Wilder [4]. It is known [4] that such a space is arc-wise connected in the strong sense (two distinct points can be connected by a homeomorph of $I$ ) and that such a space is also locally arc-wise connected. We have thus proved the

Theorem 3. A nondegenerate locally compact connected separable metrizable topological lattice is a Peano space and hence is arc-wise connected and locally arc-wise connected.

We will now specialize some of the foregoing results to the case of a topological chain.

Lemma 3. If $L$ is a connected topological chain and if $A$ is a subset of $L$ then $A$ is convex if, and only if, $A$ is connected.

Proof. If $A$ is convex and if $x$ and $y$ are elements of $A$ then, since $L$ is a chain, we have that $x \leqq y$ or $y \leqq x$. Suppose $x \leqq y$ then $x \vee(y \wedge L) \subset C(A)=A$. Now $x \vee(y \wedge L)$ is connected and so $A$ is connected. Let us now assume that $A \neq C(A)$. Since $A \subset C(A)$, we have that $C(A) \backslash A$ is nonvoid. Let $x$ be an element of $C(A) \backslash A$ then, clearly, $x \neq 0$ and $x \neq 1$. By Theorem 1 we have that $x$ cuts $L$ into exactly two components $(x \wedge L) \backslash x$ and $(x \vee L) \backslash x$. Since $A$ meets both of these sets, $A$ is not connected.

We recall that a base for the intrinsic topology of a chain (see [6]) is the collection of sets of the form $\{x \mid x<a\}$ or $\{x \mid a<x\}$ or $\{x \mid a<x<b\}$ where $a$ and $b$ are elements of the chain.

TheOREm 4. If $L$ is a connected topological chain then the following are equivalent

(i) $L$ is locally convex,

(ii) $L$ is locally connected,

(iii) the topology in $L$ is equivalent to the intrinsic topology in $L$.

(iv) $L$ is locally compact. 
Proof. The fact that (i) implies (ii) is an immediate consequence of Lemma 3. Clearly, the collection of sets of the form $\{x \mid x<a\}$ or $\{x \mid a<x\}$ or $\{x \mid a<x<b\}$ coincides with the collection of open connected sets and so we have that (ii) implies (iii). To prove that (iii) implies (iv) we rely on a result proved by Ward [7, p. 149] which implies: If $L$ is a connected topological chain endowed with its intrinsic topology and if $L$ has 0 and 1 then $L$ is compact. Now suppose that $L$ satisfies (iii) and that $a$ is an element of $L$ and that $U$ is an open set containing $a$. If $a$ is distinct from 0 and 1 then, by hypothesis, there are elements $b$ and $c$ in $L$ such that $a \in\{x \mid b<x<c\} \subset U$. By Ward's result, $\{x \mid b<x<c\}^{*}=\{x \mid b \leqq x \leqq c\}$ is compact and so $\{x \mid b<x<c\}$ is an open subset of $U$ containing $a$ whose closure is compact. If $a=0$ then there is an element $b$ in $L$ such that $\{x \mid 0 \leqq x<b\}$ is an open set containing 0 which is contained in $U$. Again $\{x \mid 0 \leqq x<b\}^{*}=\{x \mid 0 \leqq x \leqq b\}$ is compact. If $a=1$ then, by a similar argument, there is an element $b$ in $L$ such that the open set $\{x \mid b<x \leqq 1\}$ is contained in $U$ and has a compact closure. Thus $L$ is locally compact. It is known [5] that a locally compact connected topological lattice is locally convex and so (iv) implies (i) which completes the proof.

It is shown in Wilder [4] that a locally compact connected separable topological space which is irreducibly connected about two of its points is homeomorphic with $I$. Thus we have the

Corollary. If $L$ is a locally convex connected separable topological chain with 0 and 1 then $L$ is homeomorphic with $I$.

4. Simple connectivity and lattices. In this section we show that a locally compact connected subset of $R^{2}$ which admits a pair of continuous lattice operations is simply connected, i.e., the one-dimensional homotopy group of the space is trivial [9].

Lemma 4. If $L$ is a locally compact connected topological lattice and if $L$ is topologically contained in $R^{2}$ then each compact subset of $L$ has an upper bound and a lower bound in $L$.

Proof. We will establish the existence of upper bounds for compact subsets of $L$, relying on the principle of duality for the proof of the existence of lower bounds.

Property (F). An element $a$ in $L$ is said to have the property (F) if $a \in F(x \wedge L)$ for all $x \in a \bigvee L$.

Now suppose $A$ is a compact subset of $L$. If no element of $A$ has the property $(\mathrm{F})$ then for each $x \in A$ there is an element, say $y(x)$, in $x \vee L$ such that $x \in(y(x) \wedge L)^{\circ}$. Thus the family $\left\{(y(x) \wedge L)^{\circ} \mid x \in A\right\}$ is an open covering of $A$ and therefore, since $A$ is compact, there is a finite subfamily, say

$$
\left\{\left(y\left(x_{i}\right) \wedge L\right)^{\circ} \mid i=1,2, \cdots, n\right\},
$$


which cover $A$. Clearly $y\left(x_{1}\right) \bigvee y\left(x_{2}\right) \bigvee \cdots \vee y\left(x_{n}\right)$ is an upper bound for $A$. Now let us assume that some $a \in A$ has the property (F). We will show that $a \bigvee L$ is a chain. Let $x$ and $y$ be elements of $a \bigvee L$. Then $x \bigvee y$ is also in $a \bigvee L$ and so $a \in F((x \vee y) \wedge L)$. It is known [5] that if $z \in L$ and if $w \in F(z \wedge L)$ then $[w \bigvee(z \wedge L)] \subset F(z \wedge L)$. Thus letting

$$
B=a \vee[(x \vee y) \wedge L]
$$

we have that $B \subset F((x \vee y) \wedge L)$. We will now show that the codimension of $F((x \vee y) \wedge L) \leqq 1$. Let us assume that $\operatorname{cd}(F((x \vee y) \wedge L)=2$. Now $\mathrm{H}$. Cohen [3] has shown that if $X$ is a locally compact Hausdorff space then $\operatorname{cd}(X) \leqq \operatorname{ind}(X)$. Moreover, it is known [8] that if $X$ is a subset of $R^{2}$ and if $\operatorname{ind}(X)=2$ then $X$ contains a nonvoid subset which is open in $R^{2}$. It follows then that there exists a nonvoid set, $W$, which is open in $R^{2}$ with $W$ $\subset F((x \vee y) \wedge L)$. Now $W=W \cap F((x \vee y) \wedge L)=W \cap L$ and so $W$ is open in $L$. However, $(x \vee y) \wedge L$ is a closed subset of $L$ hence $F((x \vee y) \wedge L)$ is nowhere dense in $L$ which implies that $W$ is empty; but this is a contradiction. We therefore have $\operatorname{cd}(B) \leqq \operatorname{cd}(F((x \bigvee y) \wedge L) \leqq 1$. Now $B$ is a closed subset of $L$ and therefore is locally compact. Moreover, $B$ is a connected sublattice of $L$ and so $B$ is a chain (see [5]). Now $x$ and $y$ are elements of $B$ and so $x \wedge y=x$ or $y$ which implies that $a \bigvee L$ is a chain. Now $a \bigvee A$ is a compact subset of $a \bigvee L$ and so $a \bigvee A$ has an upper bound in $a \bigvee L$. Clearly, any upper bound for $a \bigvee A$ is also a upper bound for $A$.

One would suspect that this result can be proved without assuming that $L$ is topologically embedded in $R^{2}$, however the author does not know how to eliminate this hypothesis.

THEOREM 5. If $L$ is a locally compact connected topological lattice and if $L$ is topologically contained in $R^{2}$ then $\pi_{1}(L)$ is trivial.

Proof. Let $f: I \rightarrow L$ be a continuous function such that $f(0)=f(1)=x_{0}$. Since $f(I)$ is a compact subset of $L$, by Lemma 4 , there are upper and lower bounds say $a$ and $b$, for $f(I)$ in $L$. Now $a \bigvee(b \wedge L)$ is a locally compact connected sublattice of $L$ and so $a \bigvee(b \wedge L)$ is arc-wise connected. Let $g: I \rightarrow$ $a \vee(b \wedge L)$ and $h: I \rightarrow a \bigvee(b \wedge L)$ be continuous functions such that $h(0)=g(0)$ $=a, g(1)=x_{0}$ and $h(1)=b$. Define $F: I \rightarrow L$ as follows:

$$
F(t)= \begin{cases}g(3 t) & \text { if } \quad 0 \leqq t \leqq 1 / 3, \\ f(3 t-1) & \text { if } 1 / 3 \leqq t \leqq 2 / 3, \\ g(3-3 t) & \text { if } 2 / 3 \leqq t \leqq 1\end{cases}
$$

If we define $H: I \times I \rightarrow L$ by $H\left(t, t^{\prime}\right)=F(t) \wedge h\left(t^{\prime}\right)$ then clearly $H$ is continuous, $H(0, t)=H(1, t)=a, H(t, 0)=a$ and $H(t, 1)=F(t)$. Thus $F$ is homotopic to a constant mapping and so $f$ is also homotopic to a constant [9]. For further results, see [13]. 
5. The distributivity of plane lattices. In this section we show that a locally compact connected topological lattice which is topologically contained in $R^{2}$ is distributive. The proof of this result is in two parts. The first part demonstrates that such a lattice is modular and the second that it is distributive.

Lemma 6. Let $L$ be a locally compact connected topological lattice which is topologically contained in $R^{2}$. If $L$ contains four distinct elements, $a, b, m$, and $n$ such that $a \bigvee b=$ mand $a \wedge b=n$ then: (i) The set $M=[a \bigvee(m \wedge L)] \cup[a \wedge(n \bigvee L)]$ is a compact connected chain from $n$ to $m$ containing $a$ and the set $N$ $=[b \bigvee(m \wedge L)] \cup[b \wedge(n \bigvee L)]$ is a compact connected chain from $n$ to $m$ containing $b$. Moreover, $M$ and $N$ are the only connected chains from $n$ to $m$ which contain $a$ and $b$ respectively. Let $J=b \wedge M$ and let $K=b \bigvee M$. (ii) The set $M \cup N$ is a simple closed curve. (iii) $M \wedge K=[B(M \cup N)]^{*}=M \bigvee J$.

REMARK. It is known [5] that a locally compact connected topological lattice is a chain if, and only if, it is at most one-dimensional. Thus $L$ contains two unrelated elements, $a$ and $b$, if, and only if, $L$ is a two-dimensional subset of $R^{2}$.

Proof of (i). We will first prove that $m \wedge(a \vee L)$ is a chain. Since $a \ddagger b$, it follows that $m=a \bigvee b$ is in $F(a \vee L)$ (see [5]). As in the proof of Lemma 2 we have that $\operatorname{cd}(F(a \bigvee L)) \leqq 1$ and so $\operatorname{cd}(m \wedge(a \bigvee L)) \leqq 1$. Now $m \wedge(a \bigvee L)$ is a locally compact connected sublattice of $L$ and thus is a chain (see [5]). The corollary to Theorem 4 states that a locally compact connected chain with maximal and minimal elements is compact and so the set $m \wedge(a \vee L)$ is a compact connected chain from $a$ to $m$. Dually, one shows that $n \bigvee(a \wedge L)$ is a compact connected chain from $n$ to $a$ and therefore $M=[n \bigvee(a \wedge L)]$ $\cup[m \wedge(a \vee L)]$ is a compact connected chain from $n$ to $m$ containing $a$. If $C$ is any connected chain from $n$ to $m$ containing $a$ then clearly $C \subset M$. Since $M$ is irreducibly connected about $m$ and $n$ and $C$ is a connected set containing both $m$ and $n$, we have that $C=M$. In a dual fashion, one can demonstrate the existence and uniqueness of the set $N$.

Proof of (ii). Since $M$ and $N$ are each homeomorphic with the closed real number interval $I$ and $M \cap N=\{m, n\}$, the result follows immediately (see [12]).

Proof of (iii). In an effort to simplify our notation, we let $U=B(M \cup N)$ and let $V=R^{2} \backslash[U \cup M \cup N]$. The first step in the proof of this result is to establish that $U^{*} \subset M \wedge K$. Now $(M \wedge K) \wedge(M \wedge K)=M \wedge K$ and so $M \wedge K$ is a compact connected topological semigroup [10] under the operation $\wedge$. Since $n$ is a $\wedge$-zero and $m$ is a $\wedge$-unit for $M \wedge K$, we have, by a result of A. D. Wallace [11], that $H^{1}(M \wedge K)=0$. Now $M \cup N \subset M \wedge K$ and so $R^{2} \backslash(M \wedge K) \subset R^{2} \backslash(M \cup N)=U \cup V$, thus $R^{2} \backslash(M \wedge K)=[U \backslash(M \wedge K)]$ $\cup[V \backslash(M \wedge K)]$. Since $V$ is not compact and $M \wedge K$ is compact, $V \backslash(M \wedge K)$ is nonvoid. Therefore if $U \backslash(M \wedge K)$ is nonvoid then the compact connected 
set $M \wedge K$ cuts $R^{2}$ which implies that $H^{1}(M \wedge K) \neq 0$. This, however, is a contradiction and so $U \subset M \wedge K$. Since $M \wedge K$ is closed we have that $U^{*} \subset M \wedge K$. We observe that since $U^{*}$, the closure of $U$ in $L$, is compact and so $U^{*}$ is also closed in $E^{2}$ thus the closure of $U$ in $L=$ the closure of $U$ in $R^{2}=U \cup M \cup N$. We resort to the principle of duality for the proof of the fact that $U^{*} \subset M \vee J$.

Consider the function $f: M \rightarrow K$ defined by $f(x)=x \vee b$. Clearly, $f$ is continuous, order preserving and onto. Now if $x$ is an element of $K, f^{-1}(x)$ is a closed nonvoid subset of $M$, hence $\sup \left(f^{-1}(x)\right)$ exists and is contained in $f^{-1}(x)$. For each $x \in K \backslash m$, let $k(x)=\sup \left(f^{-1}(x)\right)$. Thus if $x \in K \backslash m$ then $k(x) \bigvee b$ $=x$ and $k(x)<a$. Dually, we let $j(x)=\inf \{y \in M \mid y \wedge b=x\}$ for each $x \in J \backslash n$.

The following sublemmas will be useful in proving that $M \wedge K$ and $M \vee J$ are contained in $U^{*}$.

Sublemma A. If $x \in K \backslash\{b, m\}$ then $[(k(x) \bigvee M) \wedge x] \cap[M \cup N]=\{k(x), x\}$.

Proof. Suppose $w=z \wedge x$ for some $z \in(k(x) \bigvee M)$. Since $k(x) \leqq z$ and $k(x)$ $\leqq x$, we have $k(x)<z \wedge x=w$. Now $w \bigvee x=(z \wedge x) \bigvee x=x$ and so $x=x \bigvee w$ $=(k(x) \bigvee b) \bigvee w=b \bigvee(k(x) \bigvee w)=b \bigvee w$. Thus if $w \in M$ then $w \leqq k(x)$ and therefore $w=k(x)$. Now if $w \in N$ then $w \bigvee b=b$ or $w \bigvee b=w$ and so $x=b$ or $x=w$. However $x \in K \backslash\{b, m\}$ and so $x=w$.

Dualizing this result we have

Sublemma $A^{\prime}$. If $x \in \lambda\{b, n\}$ then $[(j(x) \wedge m) \bigvee x] \cap[M \cup N]=\{j(x), x\}$.

Sublemma B. If $x \in K \backslash\{b, m\}$ then the set $[(k(x) \vee M) \wedge x] \backslash\{k(x), x\}$ is either contained in $U$ or in $V$.

Proof. Since $(k(x) \bigvee M) \wedge x$ is a connected chain from $k(x)$ to $x$, it is irreducibly connected about $k(x)$ and $x$, therefore $k(x)$ and $x$ are noncut points of $(k(x) \vee M) \wedge x$. Now by Sublemma A,

$$
[(k(x) \vee M) \wedge x] \backslash\{k(x), x\} \subset R^{2} \backslash(M \cup N)=U \cup V,
$$

hence the result follows.

Dualizing this argument we have

Sublemma $B^{\prime}$. If $x \in \lambda\{b, n\}$ then the set $[(j(x) \wedge M) \bigvee x] \backslash\{j(x), x\}$ is either contained in $U$ or in $V$.

Sublemma C. If $x \in K \backslash\{b, m\}$ and if $[(k(x) \bigvee M) \wedge x] \backslash\{k(x), x\} \subset U$ then $(k(x) \bigvee M) \wedge x=k(x) \vee J$.

Proof. Choose an element $w$ in $(k(x) \bigvee M) \wedge x$. We will show that $w \in k(x) \bigvee J$. Now $k(x)=(k(x) \bigvee n) \in k(x) \bigvee J$ and $x=(k(x) \bigvee b) \in k(x) \bigvee J$ and so if $w=x$ or $k(x)$ then $w \in k(x) \bigvee J$. If $w \neq x$ and $w \neq k(x)$ then, by hypothesis, $w \in U \subset M \vee J$. Hence there exists $y \in M$ and $z \in J$ such that $w=y \bigvee z$. Now $k(x) \leqq w \leqq x$, thus $k(x) \leqq y \bigvee z \leqq x$ and since $z \leqq b \leqq x$, we have $x=x \vee(y \vee z)$ 
$=(x \bigvee z) \bigvee y=x \bigvee y$. Now $y \in M$, therefore $y \leqq k(x)$ or $k(x) \leqq y$. If $k(x) \leqq y$ then $x=y \bigvee x=y \bigvee(k(x) \bigvee b)=(y \bigvee k(x)) \bigvee b=y \bigvee b$ which implies that $y \leqq k(x)$ and therefore $y=k(x)$ so that $w=(k(x) \bigvee z) \in k(x) \bigvee J$. If $y \leqq k(x)$ then $w=w \bigvee k(x)$ $=(y \bigvee z) \bigvee k(x)=z \bigvee(y \bigvee k(x))=z \bigvee k(x) \in k(x) \bigvee J$. We have thus established that $(k(x) \bigvee m) \wedge x \subset k(x) \bigvee J$. Now $k(x) \bigvee J$ is a connected chain from $k(x)$ to $x$, therefore, by the corollary to Theorem $1, k(x) \vee J$ is irreducibly connected about $k(x)$ and $x$. However, $(k(x) \bigvee M) \wedge x$ is a connected subset of $k(x) \vee J$ which contains both $k(x)$ and $x$, therefore $(k(x) \vee M) \wedge x=k(x) \vee J$.

The dual of Sublemma C states

Sublemma $C^{\prime}$. If $x \in J \backslash\{b, n\}$ and if $[(j(x) \wedge m) \vee x] \backslash\{j(x), x\} \subset U$ then $(j(x) \wedge M) \bigvee x=j(x) \wedge K$.

We are now in a position to prove that $M \wedge K \subset U^{*}$. We choose and fix some $c \in J$ such that $c \vee M$ meets $U$. Such an element exists since $U \subset J \vee M$. Moreover, by Sublemmas $\mathrm{B}^{\prime}$ and $\mathrm{C}^{\prime}$, we have that $c \bigvee(j(c) \wedge M)=j(c) \wedge K$. Now suppose that $x$ is an element of $K$. We will show that $x \wedge M \subset U^{*}$. Clearly, $\quad x \wedge M=[x \wedge(k(x) \vee M)] \cup[x \wedge(k(x) \wedge M)] \quad$ and $\quad x \wedge(k(x) \wedge M)$ $=k(x) \wedge M \subset M \subset U \cup M \cup N=U^{*}$. Thus it remains to show that $x \wedge(k(x) \vee M) \subset U^{*}$. If $x \wedge(k(x) \vee M) \subset M \cup N$ then $x \wedge(k(x) \vee M) \subset U^{*}$. If $x \wedge(k(x) \vee M) \nsubseteq M \cup N$ then, by Sublemma B, $[x \wedge(k(x) \bigvee M)] \backslash\{k(x), x\}$ is contained in $U$ or in $V$. Now $k(x)<a<j(c)$ and $c<b<x$ hence $j(c) \in K(x) \bigvee M$ and so $(x \wedge j(c)) \in x \wedge(k(x) \vee M)$. We also have that $(x \wedge j(c)) \in j(c) \wedge K$ $=c \vee(j(c) \wedge M)$, therefore if $x \wedge j(c) \in M$ then, by Sublemmas $\mathrm{A}$ and $\mathrm{A}^{\prime}$, $x \wedge j(c)=j(c)$ and $x \wedge j(c)=k(x)$ which is impossible. If $x \wedge j(c) \in N$ then, by Sublemmas A and $\mathrm{A}^{\prime}, x \wedge j(c)=x$ and $x \wedge j(c)=c$ which is also impossible. Thus $(x \wedge j(c)) \in[(j(c) \wedge K) \backslash\{j(c), c\}]=[c \vee(j(c) \wedge M)] \backslash\{j(c), c\} \subset U$. Consequently, $x \wedge(k(x) \bigvee M)$ meets $U$ and so $x \wedge(k(x) \bigvee M) \subset U^{*}$. A dual argument demonstrates that $J \vee M \subset U^{*}$.

A lattice is modular if for any three elements $x, y$ and $z, x \vee(y \wedge z)$ $=(x \vee y) \wedge z$ whenever $x \leqq z$. It is well known (e.g. see [6]), that a lattice is nonmodular if, and only if, it contains five distinct elements $a, b, c, m$ and $n$, such that

$$
\begin{aligned}
& \text { (a) } a \wedge c=b \wedge c=n, \\
& (\beta) a \vee c=b \vee c=m, \\
& (\gamma) a \wedge b=a .
\end{aligned}
$$

Lemma 7. Let $L$ be a topological lattice and let $a, b, c, m$ and $n$ be five distinct elements of $L$ satisfying $(\alpha),(\beta)$ and $(\gamma)$. If $M$ is a compact connected separable chain from $n$ to $m$ containing both $a$ and $b$ then $(c \wedge M) \vee M$ is not contained in $(c \vee M) \wedge M$ and $(c \vee M) \wedge M$ is not contained in $(c \wedge M) \vee M$.

Proof. Let $J=c \wedge M$ and $K=c \vee M$. As in Lemma 6, we let $k(x)$ $=\sup \{y \in M \mid y \vee c=x\}$ for $x \in K \backslash m$ and $j(x)=\inf \{y \in M \mid y \wedge c=x\}$ for 
$x \in J \backslash n$. Now suppose that $K \wedge M \subset J \bigvee M$. Since $K$ and $M$ are each homeomorphic with the closed real number interval $I$, there is a strictly monotone increasing sequence, say $\left\{x_{i}\right\}$, in $K \backslash\{m, c\}$ with $\lim \left(x_{i}\right)=m$ and a strictly monotone decreasing sequence, say $\left\{y_{i}\right\}$, in $(b \bigvee M) \backslash\{m, b\}$ with $\lim \left(y_{i}\right)=b$. Now $\wedge$ is a continuous function therefore we have that $\lim \left(y_{i} \wedge x_{i}\right)=b \wedge m$ $=b$. We will now show that for each $i=1,2,3, \cdots$ there exists an element of $J$, say $z_{i}$, such that $y_{i} \wedge x_{i}=z_{i} \bigvee k\left(x_{i}\right)$ and $z_{i} \leqq y_{i} \wedge c$. Now $y_{i} \wedge x_{i} \in K \wedge M$ $\subset J \bigvee M$ and so $y_{i} \wedge x_{i}=u \bigvee w$ for some $u \in J$ and some $w \in M$. Clearly $w \leqq u \bigvee w=y_{i} \wedge x_{i} \leqq x_{i}$ and so if $k\left(x_{i}\right) \leqq w$ then $x_{i}=w \bigvee x_{i}=w \bigvee\left(k\left(x_{i}\right) \bigvee c\right)$ $=\left(w \bigvee k\left(x_{i}\right)\right) \bigvee c=w \bigvee c$. Therefore $w \leqq k(x)$ which implies that $w=k\left(x_{i}\right)$. Now $k\left(x_{i}\right) \leqq y_{i} \wedge x_{i}$ hence if $w \leqq k\left(x_{i}\right)$ then $y_{i} \wedge x_{i}=k\left(x_{i}\right) \vee\left(y_{i} \wedge x_{i}\right)=k\left(x_{i}\right)$ $\bigvee(w \bigvee u)=\left(k\left(x_{i}\right) \bigvee w\right) \bigvee u=k\left(x_{i}\right) \bigvee u$. Also, $u \leqq c$ and $u \leqq w \bigvee u \leqq y_{i} \wedge x_{i}<y_{i}$ and so $u \leqq y_{i} \wedge c$. Thus, if we let $z_{i}=u$ then $y_{i} \wedge x_{i}=k\left(x_{i}\right) \bigvee z_{i}$ where $z_{i} \in J$ and $z_{i} \leqq y_{i} \wedge c$. Clearly, $\left\{k\left(x_{i}\right)\right\}$ is a strictly monotone increasing sequence in $M$ which is bounded above by $a$, hence $\lim \left(k\left(x_{i}\right)\right)$ exists, say $\lim \left(k\left(x_{i}\right)\right)=a_{0}$, and $a_{0} \leqq a$. Now $n \leqq z_{i} \leqq y_{i} \wedge c$ and $\lim \left(y_{i} \wedge c\right)=b \wedge c=n$, therefore $\lim \left(z_{i}\right)=n$. Thus it follows from the continuity of $\bigvee$ that $\lim \left(k\left(x_{i}\right) \bigvee z_{i}\right)=a_{0} \bigvee n=a_{0}$. We now have that $b=\lim \left(y_{i} \wedge x_{i}\right)=\lim \left(k\left(x_{i}\right) \bigvee z_{i}\right)=a_{0}$ which is a contradiction. If we assume that $J \bigvee M \subset K \wedge M$, we can dualize the foregoing argument, replacing $k(x)$ by $j(x)$, and arrive at an appropriate contradiction.

We observe that the separability of $M$ is not needed in Lemma 7. We need only to replace the notion of sequences by that of nets in this proof to eliminate this hypothesis.

THEOREM 6. If $L$ is a locally compact connected topological lattice which is topologically contained in $R^{2}$ then $L$ is modular.

Proof. Suppose $L$ is not modular, i.e. assume that $L$ contains five distinct elements, say $a, b, c, m$ and $n$, which satisfy $(\alpha),(\beta)$ and $(\gamma)$. Now $a$ and $c$ are unrelated elements of $L$ hence, by Lemma 6 , the set $M=[m \wedge(a \vee L)]$ $\cup[n \bigvee(a \wedge L)]$ is a compact connected separable chain from $n$ to $m$ containing $a$. Since $a \leqq b \leqq m, M$ also contains $b$. By Lemma 6 , we also have that $M \wedge(c \vee M)=M \vee(c \wedge M)$. However, Lemma 7 states that $M \wedge(c \vee M)$

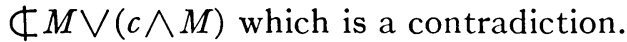

A lattice is distributive if $x \wedge(y \vee z)=(x \wedge y) \vee(x \wedge z)$ for any three elements $x, y$ and $z$. It is well known (e.g. see [7]) that a modular lattice is not distributive if, and only if, it contains five distinct elements, say $a, b, c, m$, and $n$, satisfying

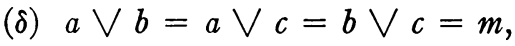

$$
\begin{aligned}
& \text { є) } a \wedge b=a \wedge c=b \wedge c=n \text {. }
\end{aligned}
$$

Theorem 7. If $L$ is a locally compact connected topological lattice which is topologically contained in $R^{2}$ then $L$ is distributive.

Proof. By the previous theorem, $L$ is modular hence if $L$ is not distribu- 
tive then $L$ contains five distinct elements, $a, b, c, m$ and $n$ which satisfy $(\delta)$ and $(\epsilon)$. Now $a, b$, and $c$ are pairwise unrelated and so, by Lemma 6 , there are unique compact connected chains from $n$ to $m$, say $A, B$ and $C$ which contain $a, b$ and $c$ respectively. Clearly, $A \cup B \cup C$ is a $\theta$-curve in the terminology of Whyburn [12] hence the bounded component of the complement of the union of two of these sets, say $A$ and $C$, contains the third set, say $B$, excepting its end points $m$ and $n$. That is to say $B \backslash\{m, n\} \subset B(A \cup C)$. Now by Lemma $3, B(A \cup C) \subset A \wedge C$, thus $b \in A \wedge C$ and so $b=x \wedge y$ for some $x \in A$ and some $y \in C$. If $y \leqq c$ then $n=b \wedge c=(b \wedge y) \wedge c=b \wedge(y \wedge c)=b \wedge y=b$ which is a contradiction. If $c \leqq y$ then $m=b \bigvee c \leqq b \bigvee y=y \leqq m$ and so $y=m$. Thus $b=x \wedge m=x \in A$ which is also a contradiction, hence $L$ is distributive.

\section{BIBLIOGRAPHY}

1. D. E. Edmondson, A nonmodular compact connected topological lattice, Proc. Amer. Math. Soc. vol. 7 (1956) p. 1157.

2. M. H. A. Newman, Elements of the topology of plane sets of points, $2 \mathrm{~d}$ ed., Cambridge, Cambridge University Press, 1951.

3. H. Cohen, A cohomological definition of dimension for locally compact Hausdorff spaces. Duke Math. J. vol. 21 (1954) pp. 209-224.

4. R. L. Wilder, Topology in manifolds. Amer. Math. Soc. Colloquium Publications, New York, 1948.

5. L. W. Anderson, $A$ note on topological lattices, Proc. Amer. Math. Soc., submitted.

6. G. Birkhoff, Lattice theory, Rev. ed., Amer. Math. Soc. Colloquium Publications, New York, 1948.

7. L. E. Ward, Jr., Partially ordered topological spaces. Proc. Amer. Math. Soc. vol. 5 (1954) pp. 144-161.

8. W. Hurewicz and H. Wallman, Dimension theory, Princeton, Princeton University Press, 1948.

9. P. J. Hilton, An introduction to homotopy theory, Cambridge, Cambridge University Press, 1953.

10. A. D. Wallace, The structure of topological semigroups, Bull. Amer. Math. Soc. vol. 61 (1955) pp. 95-112.

11. - Cohomology, dimension and mobs, Summa Brasil. Math. vol. 3 (1953) pp. 43-54.

12. G. T. Whyburn, Analytic topology, Amer. Math. Soc. Colloquium Publications, New York, 1942.

13. B. Eckmann, Räume mit Mitteldbildungen, Comment. Math. Helv. vol. 28 (1954) pp. 329-340.

TUlane University of Louisiana, New OrLeans, La. 